\title{
Dorsoventral transposition of the heart chambers in sturgeon Acipenser naccarii alevins
}

\author{
Alejandro Guerrero ${ }^{1}$, Ana C. Durán ${ }^{1}$, José M. Icardo ${ }^{2}$, Borja Fernández ${ }^{1}$, \\ Alberto Domezain ${ }^{3}$, Valentín Sans-Coma ${ }^{1, *}$ \\ ${ }^{1}$ Department of Animal Biology, Faculty of Science, University of Málaga, 29071 Málaga, Spain \\ ${ }^{2}$ Department of Anatomy and Cell Biology, University of Cantabria, 30911 Santander, Spain \\ ${ }^{3}$ Department R\&D Piscifactoría ‘Sierra Nevada', Camino de la Piscifactoría 2, 18313 Riofrío, Granada, Spain
}

\begin{abstract}
This is the first description of a dorsoventral transposition of the heart chambers in sturgeons Acipenser naccarii. The affected individuals were 2 farmed alevins aged 9 and $10 \mathrm{~d}$ posthatching, respectively. One was examined by light microscopy and the other by scanning electron microscopy. In both cases, the atrium and sinus venosus occupied a left ventrolateral position, the ventricle, conus arteriosus and bulbus arteriosus were located dorsally, and the transverse septum was incomplete. The anomalous heart examined by light microscopy did not differ histologically from normal hearts of similar developmental stages. The abnormal dorsoventral arrangement of the heart chambers was presumably due to a distortion of the morphogenetic movements that bring the ventricle to the ventral and the atrium to the dorsal position. The present findings, together with genetic data reported in the literature, suggest that the defective cardiac phenotype detected in the present specimens might result from a mutation affecting the sturgeon ortholog of the zebrafish overlooped (olp) gene.
\end{abstract}

KEY WORDS: Heart malformation $\cdot$ Cardiac chamber transposition $\cdot$ Sturgeon $\cdot$ Acipenser naccarii

\section{INTRODUCTION}

Information about cardiac anomalies in fish has notably increased in recent years, principally as a result of health surveys carried out among farmed species (see Poppe et al. 1998, 2002 for reviews, Poppe \& Taksdal 2000) and the use of the zebrafish Danio rerio as a model for the study of vertebrate heart development (Chen et al. 1996, 1997, Alexander \& Stainier 1999, Yelon \& Stainier 1999, Hu et al. 2000, Thiesse \& Zon 2002, Antkiewicz et al. 2005). In this context, a large number of cardiac malformations and dysfunctions have been reported. The aim in the present study was to describe the anatomical and histological features of a heart malformation observed for the first time in farmed alevins of the sturgeon Acipenser naccarii, an autochthonous species of the Adriatic Sea and Iberian Peninsula (GarridoRamos et al. 1997). In addition, we suggest an explanation for the present findings on the basis of current knowledge on fish heart morphogenesis.

\section{MATERIALS AND METHODS}

Samples. In an ongoing study of sturgeon Acipenser naccarii heart formation, 2 alevins were found to display a dorsoventral transposition of the cardiac chambers. One of the alevins, aged $10 \mathrm{~d}$ post-hatching (dph), was studied by means of histological techniques for light microscopy. The other, aged $9 \mathrm{dph}$, was examined by scanning electron microscopy (SEM). Ten normal alevins of the same developmental stage were used as controls. The alevins were obtained from the Sierra Nevada Fishery at Riofrío, Granada, Spain. They were overanesthetized in $0.04 \%$ MS222 (tricaine methane sulphonate; Sigma Chemicals) in fresh water.

Histological techniques for light microscopy. The alevins were fixed by immersion in MAW fixative (methanol:acetone:water, 2:2:1; ratio of fixative to tissue volume $=80: 1$ ) and embedded in Paraplast (Sigma Chemicals). Serial sections, sagittally cut at $8 \mu \mathrm{m}$, were stained with Delafield's hematoxylin-eosin (H\&E) or 
Mallory's trichrome stains. The sections were observed and photographed with a Leica DMSL light microscope.

Scanning electron microscopy. The alevins were fixed in 3\% glutaraldehyde in phosphate-buffered saline (PBS) for 3 to $5 \mathrm{~h}$. The specimens were then microdissected under a stereomicroscope, dehydrated in acetone, dried by the critical-point method using $\mathrm{CO}_{2}$ as the transitional fluid, and coated with gold. Observations were made using a Jeol JSM scanning electron microscope operated at $15 \mathrm{kV}$.

\section{RESULTS}

In order to illustrate the significance of our findings, we first describe the main anatomical and histological features of a normally developed heart in alevins aged 9 to $10 \mathrm{dph}$. At this age interval, the transverse septum is completely developed, and the anatomical arrangement of the heart basically corresponds to that of the adult sturgeon (Fig. 1). The cardiac tube is S-shaped, with a dorsally positioned atrium and a ventrally located ventricle (Fig. 1b,c). The venous pole of the heart consists of the sinus venosus that remains connected to the liver, draining the liver sinusoids, and receives the 2 lateral veins on its lateral ends. The arterial cardiac pole consists of a relatively long conus arteriosus (Fig. 1) connected to the ventral aorta through a still poorly developed bulbus arteriosus (Fig. 1a,c). Histologically, the sinus venosus and atrium are composed of a single layer of myocardiocytes, the endocardium and cardiac jelly between them. Atrium pectinate muscles are present. The atrioventricular cushions from which the atrioventricular valves develop are fully formed. The ventricle consists of an outer monolayer of myocardial cells and a set of trabeculae with the corresponding lacunary spaces. In the conus arteriosus, the proximal and distal mesenchymal cushions, which are the anlagen of the conus valves, are fully developed, showing the first signs of excavation. In the bulbus
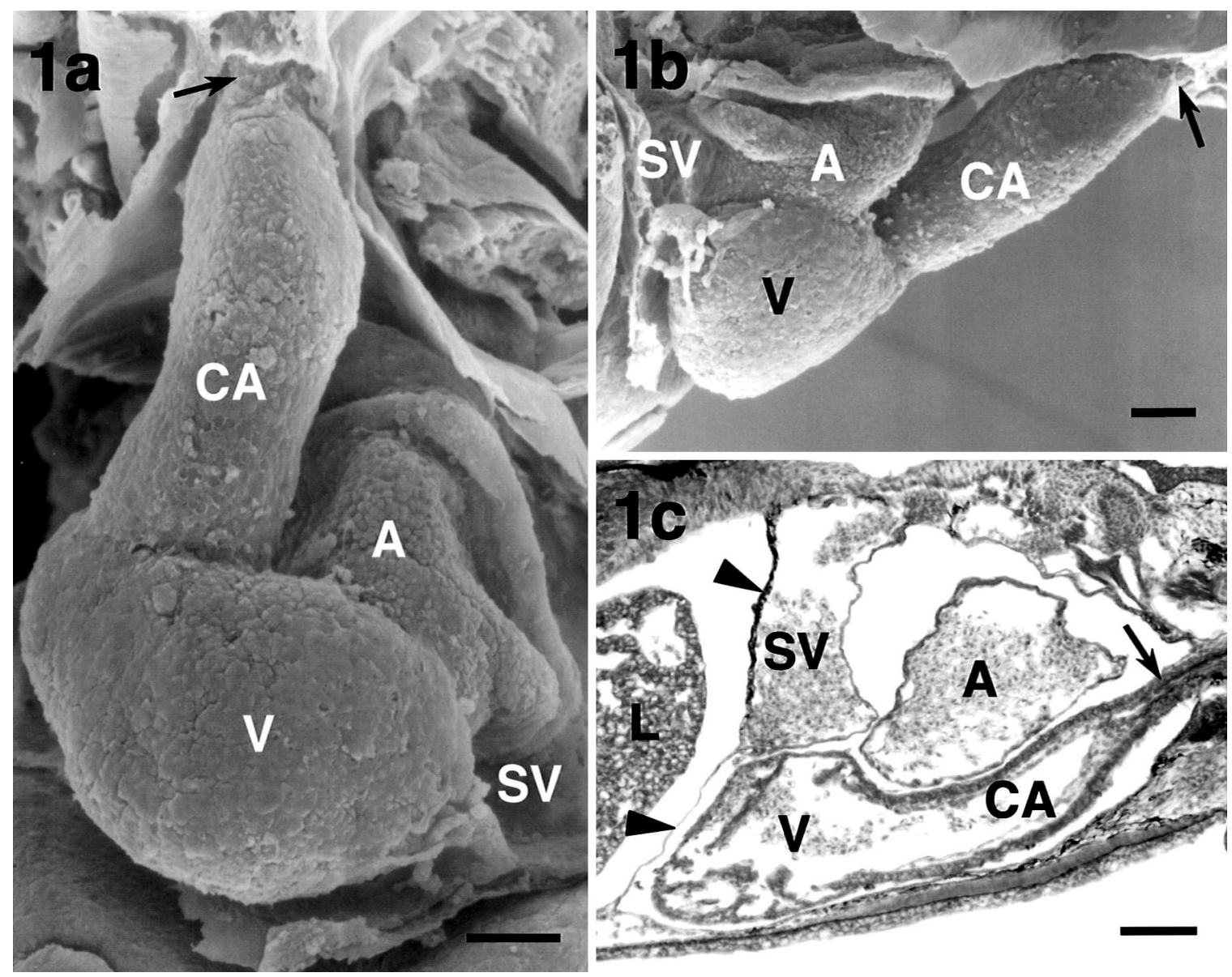

Fig. 1. Acipenser naccarii. Normally developing hearts of sturgeon alevins aged 10 d post-hatching. (a,b) Scanning electron micrographs of the same specimen in ventral (a) and right-lateral (b) views. (c) Sagittal section of the heart stained with Mallory's trichrome stain; arrowheads indicate the fully developed transverse septum. Arrows point to developing bulbus arteriosus. A: atrium; CA: conus arteriosus; L: liver; SV: sinus venosus; V: ventricle. Scale bars $=100 \mu \mathrm{m}$ 

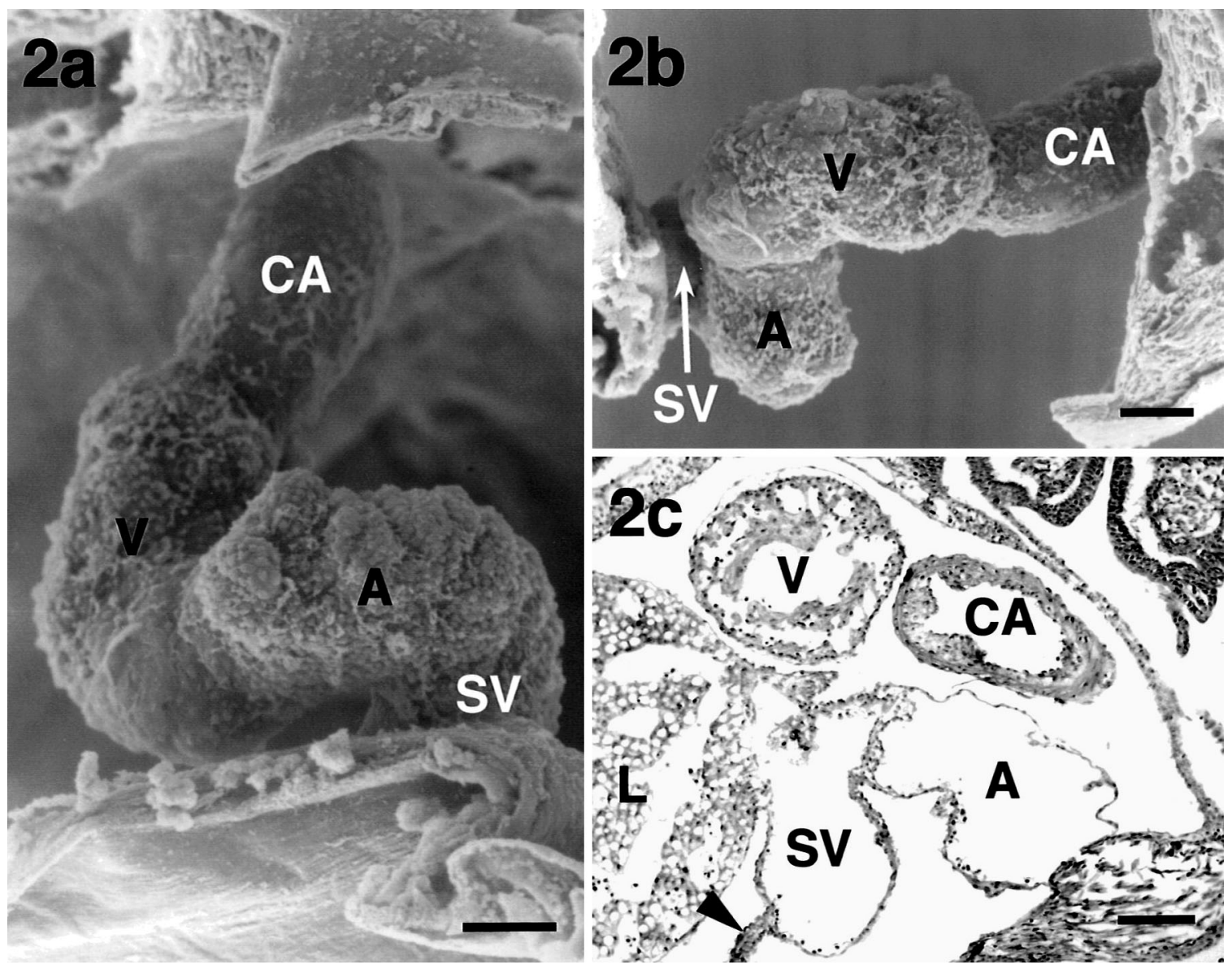

Fig. 2. Acipenser naccarii. Hearts of sturgeon alevins aged (a,b) 9 d post-hatching (dph) and (c) 10 dph, with a dorsoventral transposition of the cardiac chambers. (a,b) Scanning electron micrographs of the same specimen in ventral (a) and right-lateral (b) views. (c) Sagittal section of the heart stained with hematoxylin-eosin. The arrowhead indicates the transverse septum, which is not fully developed. A: atrium; CA: conus arteriosus; L: liver; SV: sinus venosus; V: ventricle. Scale bars $=100 \mu \mathrm{m}$

arteriosus, many cells appear aligned between the myocardium and the endocardium along the longitudinal axis. The conus arteriosus and the larger part of the ventricle are covered by the epicardium.

In the anomalous alevin aged $9 \mathrm{dph}$ (Fig. 2a,b), the transverse septum was not fully developed. All the cardiac components were somewhat reduced in size, and the heart surface showed a more irregular appearance than normal. The cardiac chambers were transposed with respect to the dorsoventral axis of the body. The sinus venosus and the atrium occupied a left ventrolateral position. The atrium extended cranially as far as the middle portion of the ventricle (Fig. 2b). The ventricle, located dorsally, was slightly shifted to the right. The conus arteriosus and the bulbus arteriosus (not shown) also occupied a dorsal position.

The other abnormal alevin, aged $10 \mathrm{dph}$, showed a complete transposition of the cardiac chambers and an incomplete transverse septum (Fig. 2c). In this case, however, the ventrally located atrium surpassed cranially the level of the conoventricular junction. The histological study revealed no difference between the abnormal and the control hearts (compare Fig. 1c with Fig. 2c).

Finally, it should be mentioned that the alevins with anomalous hearts displayed no external sign of internal anomalies. In both specimens, all the organs, with the exception of the heart, appeared normal.

\section{DISCUSSION}

The present report is the first to describe a dorsoventral transposition of the heart chambers in sturgeons Acipenser naccarii, a malformation that might be due to defective morphogenetic movements of the developing cardiac segments. In the sturgeon, cardiac looping is divided into 2 steps (Icardo et al. 2004). Prior to hatching, the heart undergoes a primary loop, during which the heart tube bends and rotates to the right, 
adopting thereby a C-shape. Around the time of hatching, the heart straightens and unloops, appearing as a tube, with the cardiac segments arranged in a caudocranial sequence: sinus venosus, atrium, ventricle and outflow tract. Thereafter, during the first $4 \mathrm{dph}$, a secondary loop forms that brings the heart back to a Cshape. This is followed by a counterclockwise rotation that moves the atrium to the left of the outflow tract and the ventricle to a caudal position. From 5 to $6 \mathrm{dph}$, the atrium moves cranially and part of it passes dorsally to the outflow tract, appearing on its right side. Meanwhile, the sinus venosus shifts from a caudal to a dorsal position, and the ventricle becomes located ventrally, along the body midline. In addition, the arterial pole of the heart differentiates into a proximal, myocardial conus arteriosus and a distal, non-myocardial bulbus arteriosus (Guerrero et al. 2004).

In the anomalous sturgeon hearts in the present study, the cardiac segments showed a normal arrangement with regard to the left-right body axis. In addition, the heart had acquired its characteristic C-shape, indicating that the secondary loop had formed normally. This suggests that the anomalous heart condition resulted from a distortion of the morphogenetic movements which, after looping, bring the atrium and cardiac venous pole to the dorsal position, and the ventricle and cardiac arterial pole to the ventral position.

A cardiac malformation similar, in part, to that of the alevins in our study, was reported by Poppe et al. (2002) in adult farmed rainbow trout Oncorhynchus mykiss. In the affected specimens, the ventricle and the bulbus arteriosus were located dextrodorsally with respect to the atrium. However, in contrast to the sturgeon alevins, the sinus venosus and the atrium of the rainbow trout were notably enlarged. The atrium displayed a saccular shape and protruded through the muscles of the ventral body wall, forming a hernia covered by the dermis and the epidermis. The outer compact layer of the ventricular myocardium was hypoplastic. All the other rainbow trout organs were normal, as was the case in the sturgeon alevins. The etiology of the cardiac defects found in the rainbow trout could not be determined. Environmental and hereditary factors were suggested as possible causative factors. However, it was considered that the cardiac hernia was most likely the result of malfunction of the ventricle, leading to congestion with resultant dilatation of the atrium, which, in turn, had pushed the hypoplastic ventricle away.

Poppe et al. (1998) described 2 adult specimens of farmed Atlantic salmon Salmo salar in which the heart was rotated counterclockwise on its transverse axis. The ventricle was elongated, and the apex of the heart pointed dorsally. The atrium was situated under the ventricle within a normal pericardial cavity. In these cases, genetic factors were also suggested as causing the heart abnormalities (Poppe et al. 1998).

In the last decade, numerous mutations affecting the cardiovascular system of the zebrafish Danio rerio have been identified (Chen et al. 1996, Stainier et al. 1996, Alexander \& Stainier 1999). Several of them concern cardiac morphogenesis, generating a wide variety of anomalous phenotypes. To our knowledge, the only mutation recorded to date that affects the dorsoventral arrangement of the cardiac chambers was found in the gene overlooped (olp) (Chen et al. 1996). In the olp mutants aged $2 \mathrm{~d}$, the ventricle is located on the top of the atrium, at a $90^{\circ}$ angle, instead of parallel to the atrium. On this basis, we presume that the heart chamber transposition observed in the present sturgeon alevins, and perhaps the analogous cardiac malformation found in the salmon (Poppe et al. 1998), might result from a mutation affecting their respective orthologs of the zebrafish olp-gene.

It should be underscored that the dorsoventral transposition of the heart chambers in the sturgeon was accompanied by a deficient development of the transverse septum. Although the etiologic relationship between both anomalous conditions is uncertain, the existence of some common link cannot be ruled out. Deficiencies of the transverse septum associated with heart malformations were reported in adult farmed Atlantic salmon Salmo salar (Poppe et al. 1998). In those cases, however, the heart was either located along the left abdominal wall or distorted counterclockwise, with the apex pointing in a dorsal or dorsocaudal direction.

To what extent the cardiac anomaly found in the present sturgeon alevins might have compromised their survival remains an open question. In this context, it should be noted that the cardiac outflow tract of the olp zebrafish mutants became slightly enlarged after $3 \mathrm{~d}$ of development (Chen et al. 1996). However, the contractility of the heart was found to be relatively normal compared to that of its wild-type siblings, a fact that led to the conclusion that there is no reason to presuppose that the zebrafish olp-homozygotes could not survive until adulthood.

Acknowledgements. This work was supported by Grants CGL2004-06306-CO2-01/BOS and CGL2006-00693 from the D.G.I. (Ministerio de Educación y Ciencia, Spain). The authors express their deep gratitude to G. Martín, Málaga, for operating the scanning electron microscope.

\section{LITERATURE CITED}

Alexander J, Stainier DYR (1999) Mutations affecting cardiac development in zebrafish: In: Harvey RP, Rosenthal N (eds) Heart development. Academic Press, San Diego, CA, p 91-110 
Antkiewicz DS, Burns CG, Carney SA, Peterson RE, Heideman W (2005) Heart malformation is an early response to TCDD in embryonic zebrafish. Toxicol Sci 84:368-377

Chen JN, Haffter P, Odenthal J, Vogelsang E and others (1996) Mutations affecting the cardiovascular system and other internal organs in zebrafish. Development 123:293-302

Chen JN, van Eeden FJM, Warren KS, Chin A, NüssleinVolhard C, Haffter P, Fishman MC (1997) Left-right pattern of cardiac BMP4 may drive asymmetry of the heart in zebrafish. Development 124:4373-4382

Garrido-Ramos M, Soriguer MC, De la Herrán R, Jamilena M and others (1997) Morphometric and genetic analysis as proof of the existence of two sturgeon species in the Guadalquivir river. Mar Biol 129:33-39

Guerrero A, Icardo JM, Durán AC, Gallego A, Domezain A, Colvee E, Sans-Coma V (2004) Differentiation of the cardiac outflow tract components in alevins of the sturgeon Acipenser naccarii (Osteichthyes, Acipenseriformes): implications for heart evolution. J Morphol 260: $172-183$

Hu N, Sedmera D, Yost HJ, Clark EB (2000) Structure and function of the developing zebrafish heart. Anat Rec 260: 148-157

Editorial responsibility: Stewart Johnson,

Halifax, Nova Scotia, Canada
Icardo JM, Guerrero A, Durán AC, Domezain A, Colvee E, Sans-Coma V (2004) The development of the sturgeon heart. Anat Embryol 208:439-449

Poppe TT, Taksdal T (2000) Ventricular hypoplasia in farmed Atlantic salmon Salmo salar. Dis Aquat Org 42: $35-40$

Poppe TT, Midtlyng P, Sand RD (1998) Examination of abdominal organs and diagnosis of deficient septum transversum in Atlantic salmon, Salmo salar L., using diagnostic ultrasound imaging. J Fish Dis 21:67-72

Poppe TT, Johansen R, Torud B (2002) Cardiac abnormality with associated hernia in farmed rainbow trout Oncorhynchus mykiss. Dis Aquat Org 50:153-155

Stainier DYR, Fouquet B, Chen JN, Warren KS and others (1996) Mutations affecting the formation and function of the cardiovascular system in the zebrafish embryo. Development 123:285-292

Thiesse C, Zon L (2002) Organogenesis-heart and blood formation from the zebrafish point of view. Science 295: $457-461$

Yelon D, Stainier DYR (1999) Patterning during organogenesis: genetic analysis of cardiac chamber formation. Cell Dev Biol 10:93-98

Submitted: April 30, 2007; Accepted: August 3, 2007 Proofs received from author(s): November 14, 2007 